\title{
The Wireless Environment Monitoring Alarm System Based on Self- organizing Network
}

\author{
Zhang Huawei $^{1, *}$, Li Nan $^{2}$, Jiang Zhanjun ${ }^{1}$ and Li Qiang ${ }^{1}$ \\ ${ }^{I}$ School of Electronic and Information Engineering, Lanzhou Jiao Tong University, Lanzhou, Gansu, 730070, China \\ ${ }^{2}$ The Experiment Central of the Computer Science and Technology, Lanzhou Jiao Tong University, Lanzhou, Gansu, \\ 730070, China
}

\begin{abstract}
Under complicated conditions, it is necessary for environmental monitoring to design a wireless monitoring alarm system which can replace the wired system or as a supplement. The system discussed here bases on ARM7 microprocessor named LPC1114 and transceiver module named CC2530. With ZigBee, CSM/GPRS, this system uses multiple sensors to self-organized form a data acquisition and monitoring network system with variety of sensors fusion in the region. The system has some characteristics such as quick, convenient and accurate. Combining with the GSM SMS or GPRS alarm, the system can accurately and reliably monitor temperature, humidity and other environmental factors, and realize remote monitoring in large area and the complicated environment. Thus, this system has high practical value.
\end{abstract}

Keywords: ARM, Self-organizing network, Wireless environment monitoring, ZigBee.

\section{INTRODUCTION}

With the rapid development of economy and the unceasing enhancement of social modernization, the environment monitoring has attained an important role in various industries. Environmental monitoring can be divided into two parts; the wired environmental monitoring and the wireless environment monitoring.

The wireless environment monitoring alarm system is wireless, it is easy to install and has a quick response. It monitors, perceives and gathers various detection object information in real-time which belongs to the regional environment of network, transmitting these information to the user or management platform for processing. The wireless environment monitoring alarm system is widely used in residential quarters, public places, office buildings and warehouses. The multi-node, more jump, peer-to-peer and wireless self-organized network (mobile AD hoc network) system is especially suitable for some areas where people cannot safely enter into, such as inflammable and explosive dangerous sites, as well as some places that cable network can not reach, such as forest, grassland, remote areas, etc. [1].

\section{ENVIRONMENTAL MONITORING SYSTEM DE- SIGN}

The system is composed of the main console of ARM and the ZigBee sensor network. ZigBee sensor network collects data and timely feedbacks to the main console, the main console can display the current position distribution and status information from each terminal node, processing data, alarming the abnormal data. If there is no staff at the scene, it will inform administrator via the GSM network remotely, and then handle abnormal data in a timely manner. The design block diagram about whole system is shown as Fig. (1).

\subsection{Main Console Hardware Design}

The main console is composed of LPC1114 microprocessor, keyboard, 12864 LCD module, AT24C02 storage module, ISD1420 voice module and buzzer alarm module [2].

LPC1114 is produced by NXP, and it is the world's most popular 32-bit ARM Cortex-M0 microprocessor, using 32 KB Flash and 8 KB SRAM. Comparing with the existing $8 / 16$ bits MCU, it has the advantages of high cost performance, low power consumption, simple instruction set and small code length.

ISD1420 is a single chip voice recording circuit, which is composed of the oscillator, voice storage unit, preamplifier, automatic gain control circuit, anti-jamming filter and output amplifier. It is the alarm sound module of information output in the system. While alarming happens, the recorded alarm sound will be read under the action of low level pulse.

NXP LPC1114 is the key of Main console, connecting ZigBee coordinator via the UART serial port for data exchange.

As the system begins to work, ISD1420 voice chip is used to play a prompt voice of welcome, 12864 liquid crystal display module can display sensor data which the main 
Table 1. Partial information data collected by Temperature and humidity sensors.

\begin{tabular}{|c|c|c|c|c|c|c|c|}
\hline Temperature $\left({ }^{\circ} \mathbf{C}\right)$ & $\mathbf{1 6 . 3}$ & $\mathbf{2 4 . 7}$ & $\mathbf{3 0 . 5}$ & Humidity (\%RH) & $\mathbf{2 9 . 5}$ & $\mathbf{3 4 . 4}$ & $\mathbf{4 0 . 4}$ \\
\hline \hline Node 1 & 16.5 & 24.9 & 30.8 & node 1 & 31.2 & 35.6 & 42.1 \\
\hline Node 2 & 16.5 & 24.8 & 38.6 & node 2 & 28.7 & 32.9 & 39.2 \\
\hline Node 3 & 16.3 & 24.8 & 30.4 & node 3 & 30.1 & 34.9 & 41.6 \\
\hline Node 4 & 16.4 & 24.8 & 30.6 & node 4 & 29.3 & 34.1 & 40.3 \\
\hline
\end{tabular}

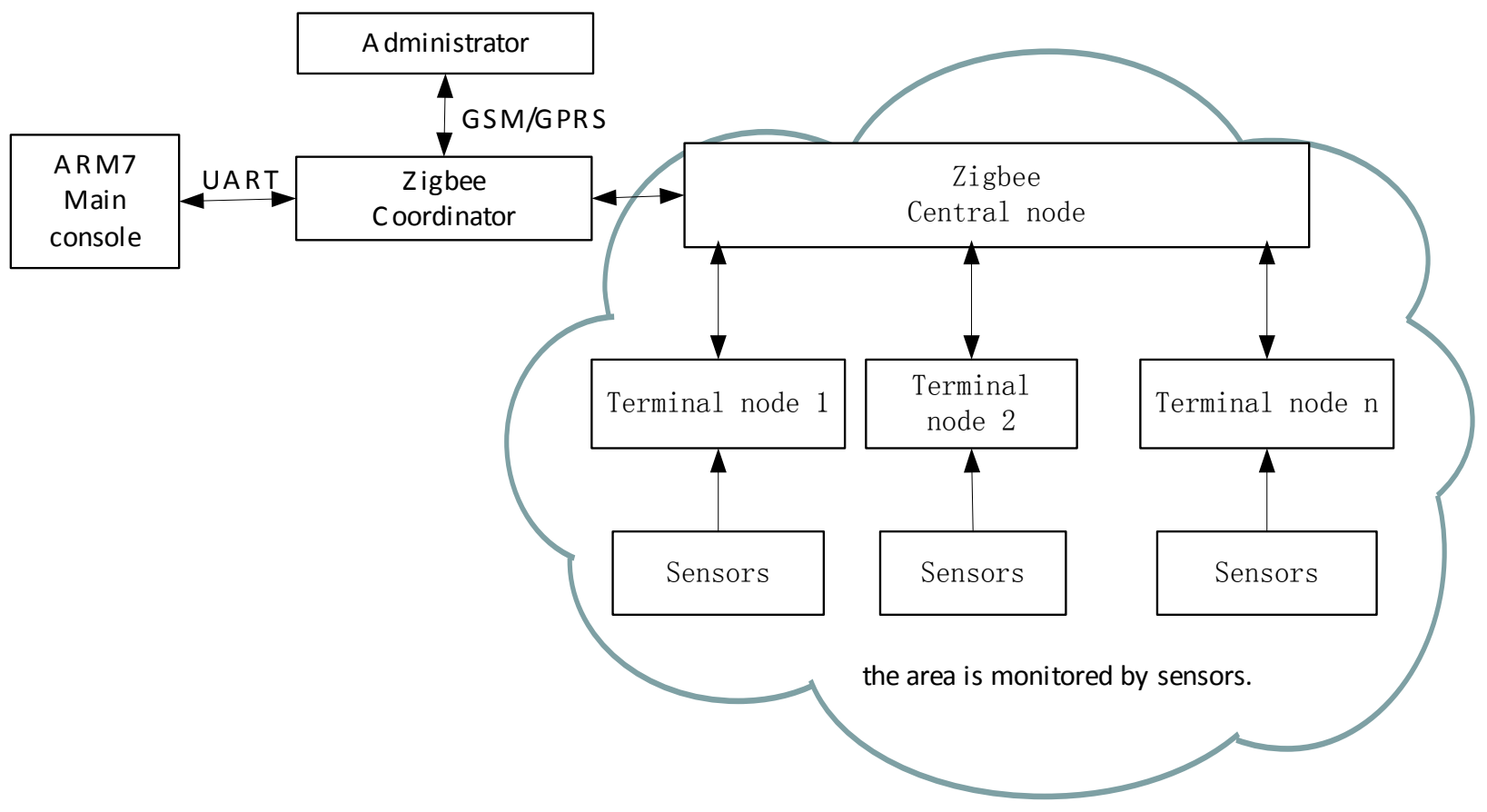

Fig. (1). The structure of wireless environment monitoring alarm system.

console receives by menu. After the system is initialized, LCD screen displays the current time and the temperature of the current environment. With the key module the time can be changed, upper and lower threshold values of the sensor data will be set $[3,4]$. Considering the instantaneity and reliability of system, power module section is done with intelligent design. While the main power supply is broken, the system will automatically use spare power. The main console hardware design is shown as Fig. (2).

\subsection{ZigBee Sensor Network Design}

ZigBee is widely used now as a wireless network transmission technology. It uses the IEEE802.15.4 protocol standard which uses public frequency $2.4 \mathrm{GHz}$ of global sharing. ZigBee wireless indoor transmission distance is 30$50 \mathrm{~m}$ and can be raised up to $100 \mathrm{~m}$ outdoor. As a result, according to change of the circumstance, the user will form a short distance wireless communication subnet, using wireless transceiver to transmit data quickly and easily. It does not only reduce the network cabling cost, but also improve the flexibility and extensibility of the application, and economize human resources [5].

The wireless sensor network apply a star topology, includes ZigBee coordinator, ZigBee central node, ZigBee terminal nodes, and each sensor node responsible for collecting data. No matter what kind of network topology is adopted, the coordinator node can have the only one, which is located in the upper layer of sensor nodes, acts as a gateway, as a whole to monitor and manage all the devices in the wireless sensor network. In monitoring area, a large amount of sensors are placed, constituting a monitoring area network by wireless self-organized way, timely collecting data by ZigBee terminal nodes to upload to the ZigBee central node. According to the agreed scope of data, central node determines whether the received data is normal and displays data through the liquid crystal or not. If there is any abnormal data, it will send alarm information to the console, during the prescribed period if the abnormal information has not been processed and is resend. After be sent three times, if central 


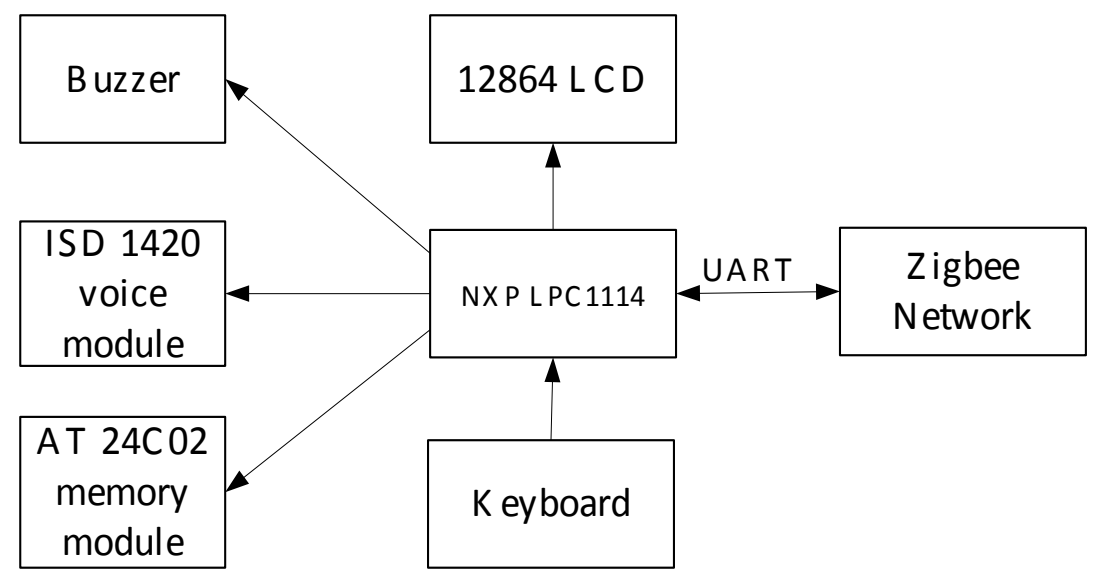

Fig. (2). The design of main console based on ARM.

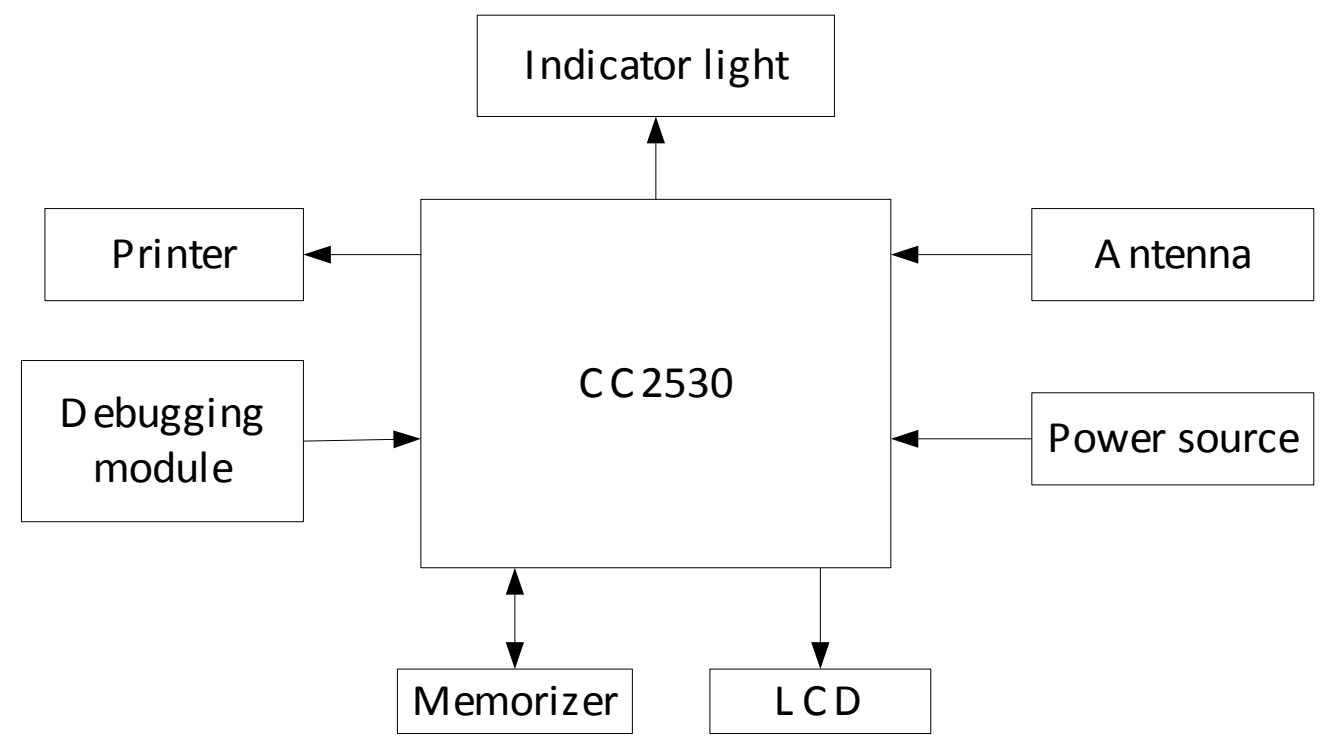

Fig. (3). The hardware design of ZigBee coordinator node.

node has not received the processing message, it can send SMS (phone) by GSM/GPRS module to the predefined staff's phone number [6-8]. This system should possess the following sensors driver according to the needs as follows: DHT90 temperature and humidity sensor, smoke sensor, CO sensor, the human body infrared sensor, etc.

\subsection{ZigBee Coordinator Nodes' Hardware Design}

ZigBee coordinator node is the rule maker for the whole sensor network. The hardware design block diagram is shown as Fig. (3). The coordinator node can take the initiative to scan all the sensors of coverage areas in the wireless network, and be responsible for receiving data from sensors, transmitting to the main console via a serial port, connecting ZigBee sensor network, and completing the node joining and deleting as well as the conversion of network protocol and so on.
CC2530 chip is a complete $2.4 \mathrm{GHz}$ radio frequency (RF) solutions on a chip system launched by TI company, is integrated with $2.4 \mathrm{GHz}$ direct sequence spread spectrum RF transceiver, industrial-grade enhanced 8051 microprocessor cores, up to $256 \mathrm{~KB}$ flash memory, 8KB RAM, 12 bit A/D converter of 8 channel, two UART interface, 21 GPIO, and so on. CC2530 chip has four kinds of power supply mode. It has high wireless receiver sensitivity and anti-interference, transmitting distance is more than $75 \mathrm{~m}$, the highest transfer rate is $250 \mathrm{Kbps}$, which simplifies the $\mathrm{RF}$ circuit design greatly. CC2530' $\mathrm{s}$ work temperature is $-40{ }^{\circ} \mathrm{C}-+125^{\circ} \mathrm{C}$ and its work voltage is from $2.0 \mathrm{~V}$ to $3.6 \mathrm{~V}$. During hibernation, the power consumption can be reduced to $0.6 \mathrm{~A}$. CC2530 has low power consumption and good adaptability in the environment [9].

ZigBee coordinator node is equipped with a rod module, which is crucial for data transmission; Power supply module 


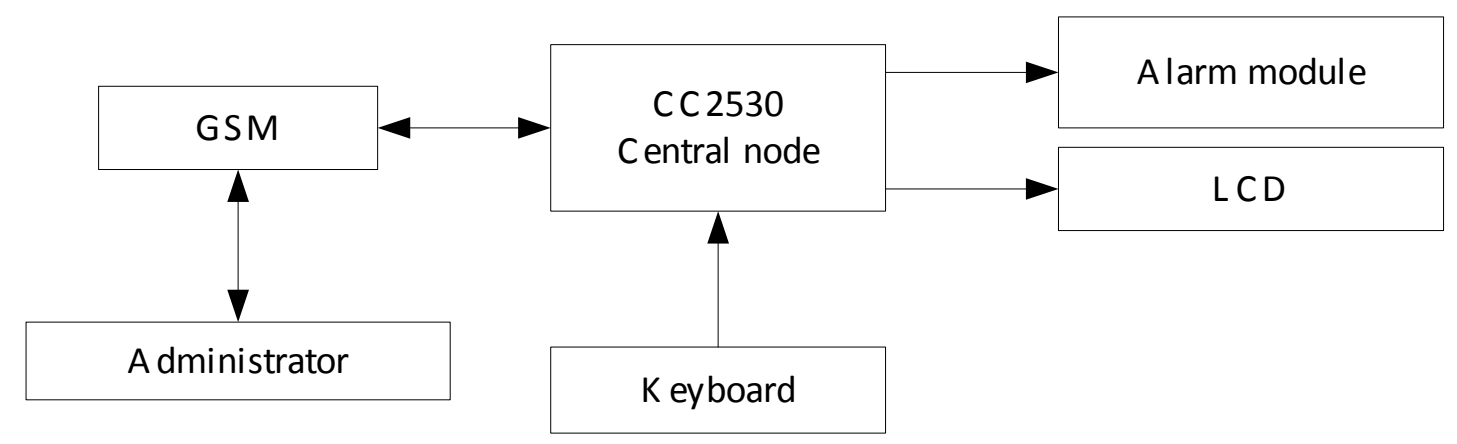

Fig. (4a). Hardware design of ZigBee central node.

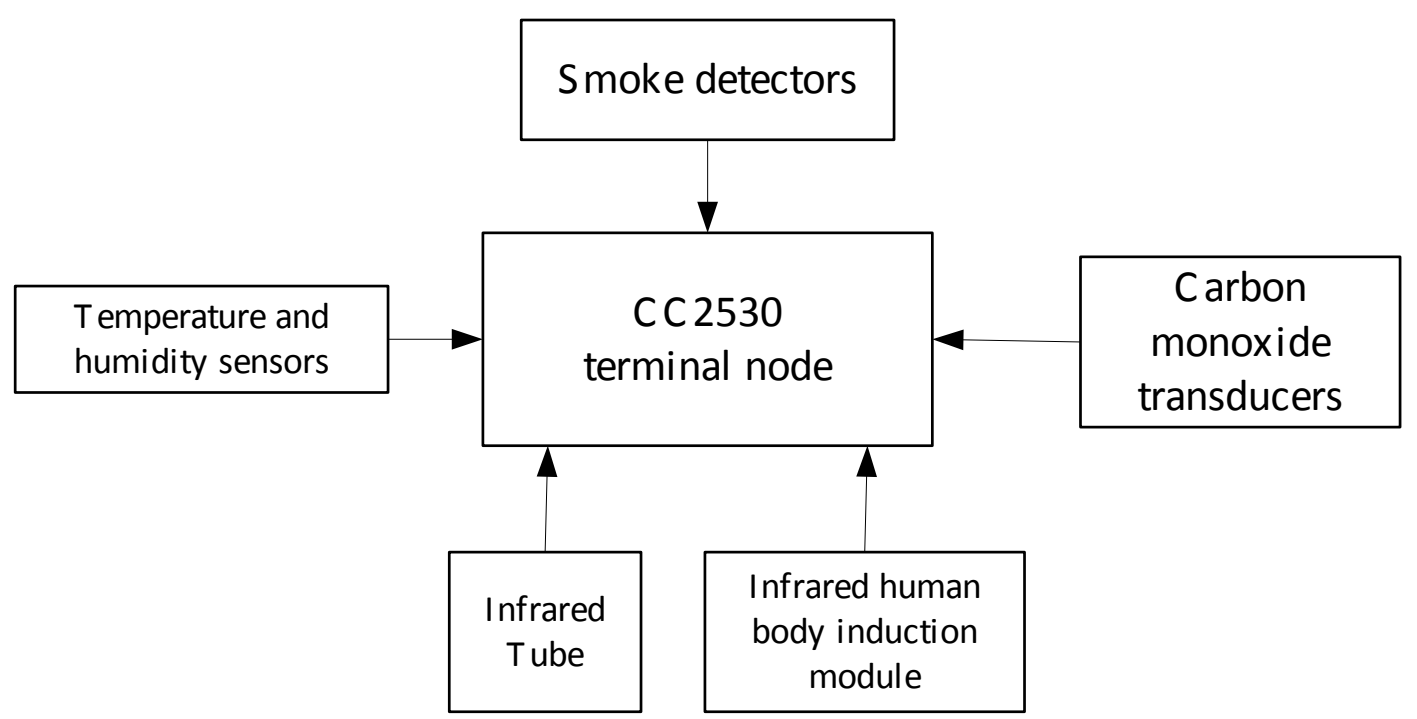

Fig. (4b). Hardware design of ZigBee terminal node.

is $5 \mathrm{~V}$ power supply, which is transformed to $3.3 \mathrm{~V}$ by TPS73033 and MIC29302 chip, supplying the power to a CC2530 chip. Liquid crystal display module displays each terminal sensor signals. Memory module backup necessary data in order to provide check for the administrators [10].

\subsection{ZigBee Central Node and Terminal Node Hardware Design}

ZigBee central node is located between ZigBee coordinator and ZigBee terminal nodes. It is necessary to monitor environmental parameters around, but also as a transit point to support communication between $\mathrm{ZigBee}$ central node and ZigBee terminal nodes. Moreover, ZigBee central node is connected to the GSM module and will timely send an abnormal message to the phone of the administrator to ensure that administrators have the real-time understanding of abnormal information in the case of the main console unattended.

ZigBee terminal devices are tiny nodes running on battery, taking own sensor be responsible for collecting environmental data around, regularly uploads data to ZigBee central node. The block diagrams of ZigBee central node and terminal node hardware design are shown as Figs. (4a and $\mathbf{b})$.

\section{SYSTEM SOFTWARE DESIGN}

The system software design is composed of two parts which are the main console software design and ZigBee node software design. ZigBee node software design is divided into ZigBee terminal nodes for collecting data, ZigBee central node for forwarding the data and ZigBee coordinator node for receiving the data. The followings only describe the software design of ZigBee coordinator node.

\subsection{Main Console Software Design}

LPC 1114 microprocessor is embedded by Linux operating system as a support, adopting MDK + ULINK2 as compilers and emulators, writing the main program and interactive interface of the main console in QT / Embedded environment, using JTAG interface of the LPC1114 to easily debug program and download code through emulator ULINK2, and realizeing system functions ultimately.

It is primary that main console is started, system is initialized and time and temperature are displayed. Through the keys, time can be adjusted, the temperature alarm thresholds are set and all node data are checked. CC2530 forwards data ZigBee terminal node transmitting to the main console. 


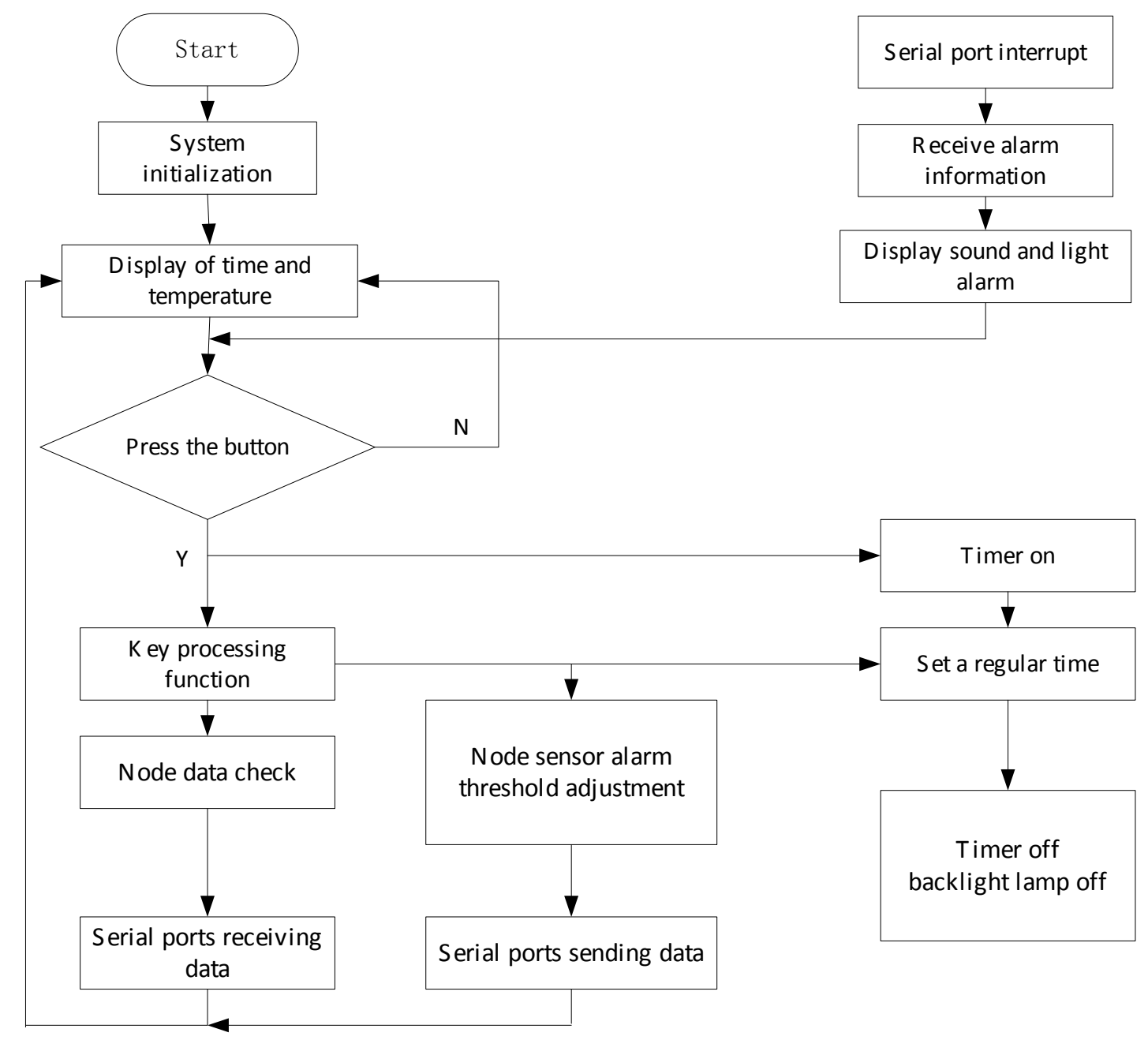

Fig. (5). The software diagram of main console.

The block diagram of main console program design is shown as Fig. (5).

\subsection{ZigBee Coordinator Software Design}

After ZigBee coordinator starts, ZigBee coordinator sends a network message to the central node. If the networking is successful, ZigBee coordinator waits for forwarding data of the main console or ZigBee central node, transmitting a confirm information, opening timer, and then waits for a reply. In the event of timeout, retransmitting will happen. Its specific software flow chart is shown as Fig. (6).

\section{SYSTEM PERFORMANCE TESTING AND RE- SULTS}

The wireless environmental monitoring system is tested from the reliability, transmission distance and real-time performance of data. Firstly, temperature values collected and air-conditioning temperature values displayed are basically the same. The error does not exceed $0.5^{\circ} \mathrm{C}$ when the node is placed conditioned room. Obviously, the deviation is in the allowable range. Humidity difference is $3 \% \mathrm{RH}$, some of the testing data are shown as Table 1. On the top, it is installed the human body infrared sensor outside the door. The sidemounted is reflection infrared sensor, which makes accuracy reach more than $99 \%$ in the day and at the night. The alarm accuracy rate of sensitivity of $\mathrm{CO}$ sensors and smoke sensor at room temperature is more than $99 \%$. When the main console is unattended, after one minute, GSM short message is successfully sent (1000 tests). Secondly, the transmission distance of actual measured data is about 300 meters in case of accessibility, it reaches about 100 meters in case there is an obstacle. In addition, two nodes are placed in different places of the open outdoors, where the distance between two nodes is in the range of the wireless sensor network (the distance between two nodes is $200 \mathrm{~m}$ ). The nodes can receive data of all nodes that send packets in about 20 seconds. The difference is in the allowable range. The test results above show that wireless environment monitoring alarm system designed in this paper can complete the intended functions. 


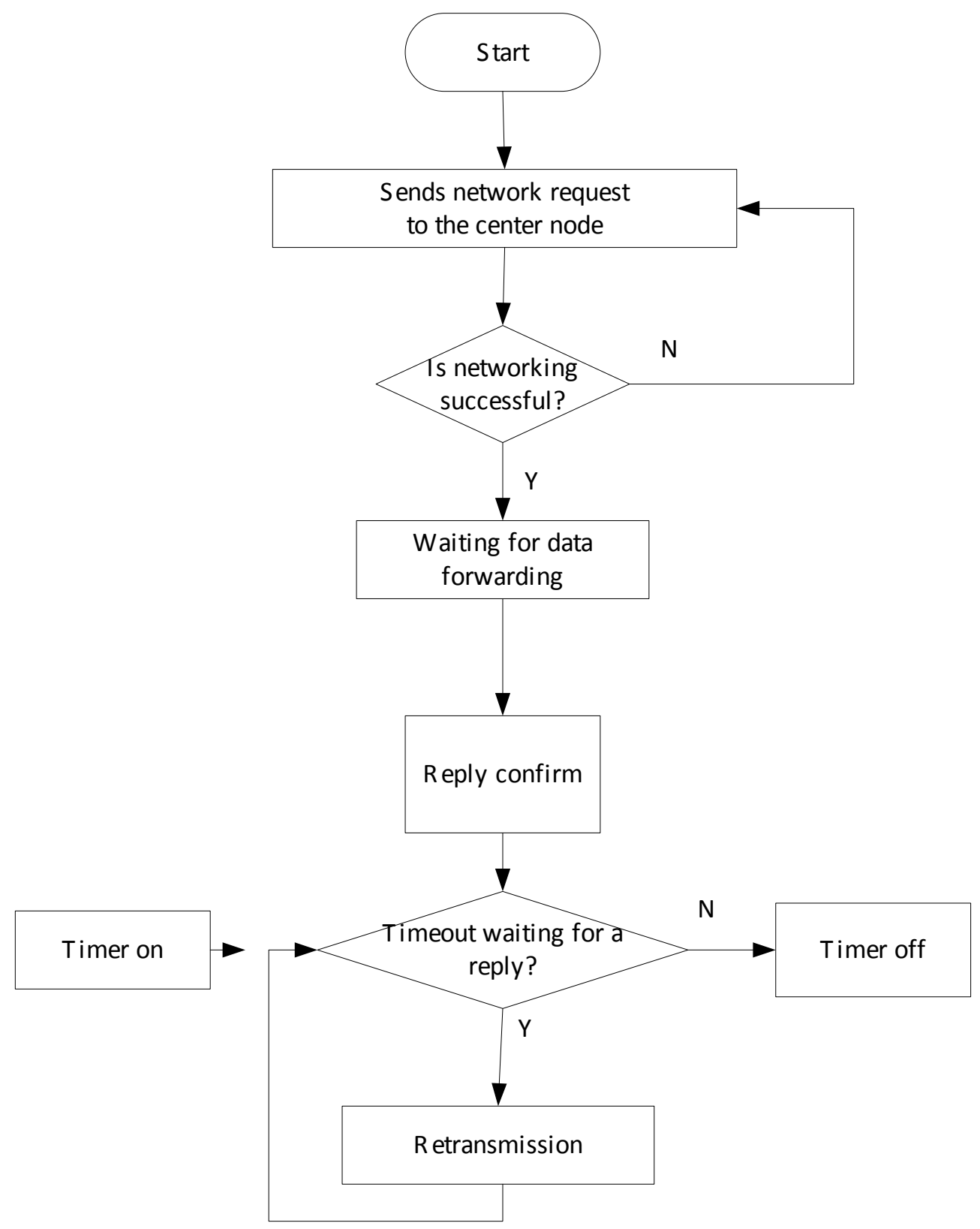

Fig. (6). The software diagram of ZigBee coordinator node.

\section{CONCLUSION}

This paper presents a design and implementation scheme of wireless sensor networks which can be used in the harsh environment monitoring. It uses low-power ARM7 microprocessor LPC1114 as the main control console, consists of a variety of sensor by the ZigBee, CSM/GPRS, forming a multi sensor fusion of the data acquisition and monitoring network system. The system overcomes the bottleneck of traditional transmission rate of wireless sensor network. The ZigBee nodes in the system are powered by battery, so each monitoring point will sent data to the main control console when it receives command of the main control console or monitors the environment abnormality, or, it will not send data. So it reduces the energy consumption of the power supply. The main console can receives the ZigBee terminal nodes' data in real time and check the monitoring area sen- sors' data at any time. According to the practical running tests, it shows that this system has some characteristics such as high reliability, low cost, strong adaptability, easy expansion and high practical value.

\section{CONFLICT OF INTEREST}

The authors confirm that this article content has no conflict of interest.

\section{ACKNOWLEDGEMENTS}

I would like to express my gratitude to all those who helped me during the writing of this thesis. My deepest gratitude goes first and foremost to Professor Jiang Zhanjun, for his instructive advice and useful suggestions on my thesis. 
This work is supported by the Gansu natural science foundation (145RJZA206) and the LanZhou JiaoTong University Youth Science Fund (2012005).

\section{REFERENCES}

[1] K. Ishida, "The present status and development trends of environmental monitoring," Environmental Monitoring in China, vol. 1, pp. $4-7,2005$.

[2] L. Tang, X. Zheng, X. Su, S. Zheng, and G. Shao, "Experimental mobile environmental monitoring and real-time analysis as an initial application of EIoT in town villages in China," In: International Journal of Sustainable Development \& World Ecology, vol. 20, no. 3, pp. 267-271, 2013.

[3] M. Martinelli, D. Moroni, G. Pieri, and M. Tampucci, "A proactive gis infrastructure for environmental monitoring," In: Seventeenth International Water Technology Conference (IWTC17), Nov. 2013.

[4] X. Li, L. Xu, H. Wang, J. Song, and S.X. Yang, "A differential evolution-based routing algorithm for environmental monitoring wireless sensor networks," Sensors, vol. 10, no. 6, p. 5425, 2010.
[5] H. Wang, S. Shen, "Design and research of internet of things in environmental monitoring based on ZigBee," Hans Journal of Wireless Communications, vol. 3, no. 5, pp. 115-121, 2013.

[6] J. Guevara, F. Barrero, E. Vargas, J. Becerra, and S. Toral, "Environmental wireless sensor network for road traffic applications," IET Intelligent Transport Systems, vol. 6, no. 2, pp. 177-186, 2012.

[7] X. Du, X. Lu, and X. Bai, "Research on intelligent energy saving system based on Zigbee and ARM," Journal of Taiyuan University of Technology, vol. 43, no. 6, pp. 762-765, 2012.

[8] H. Liu, and L. Cheng, "Design of environmental mornitoring system with mobile sink," Computer Engineering and Applications, vol. 46. pp. 7-9, 2010.

[9] L.M.P. De Brito, L.M.R. Peralta, F.E.S. and Santos, R.P.R. Fernandes, "Wireless sensor networks applied to museums environmental monitoring," In: The $4^{\text {th }}$ International Conference on Wireless and Mobile Communications ICWMC, 2008, pp. 364-369, 2008.

[10] P. Hong, and M. Jin, "Following Design Things and efficient environmental monitoring system based on LabVIEW sensors and micro-systems, vol. 32, no. 2, pp. 128-130, 2013.

(C) Huawei et al.; Licensee Bentham Open.

This is an open access article licensed under the terms of the Creative Commons Attribution Non-Commercial License (http://creativecommons.org/licenses/bync/3.0/) which permits unrestricted, non-commercial use, distribution and reproduction in any medium, provided the work is properly cited. 